\title{
The 8-GeV Transfer Line Injection into Main Ring
}

\author{
Ming-Jen Yang \\ Fermi National Accelerator Laboratory \\ P.O. Box 500, Batavia, Illinois 60510
}

June 1995 


\section{Disclaimer}

This report was prepared as an account of work sponsored by an agency of the United States Government. Neither the United States Government nor any agency thereof, nor any of their employees, makes any warranty, express or implied, or assumes any legal liability or responsibility for the accuracy, completeness, or usefulness of any information, apparatus, product, or process disclosed, or represents that its use would not infringe privately owned rights. Reference herein to any specific commercial product, process, or service by trade name, trademark, manufacturer, or otherwise, does not necessarily constitute or imply its endorsement, recommendation, or favoring by the United States Government or any agency thereof. The views and opinions of authors expressed herein do not necessarily state or reflect those of the United States Government or any agency thereof. 
TM -1923

\title{
The 8-GeV Transfer Line Injection into MainRing
}

\author{
Ming-Jen Yang \\ Fermi National Accelerator Laboratory \\ P.O. Box 500, Batavia, Illinois \\ March 1995
}




\section{Table of content}

1. Introduction .......................................................................................... 1

2. Design lattice function .......................................................................... 1

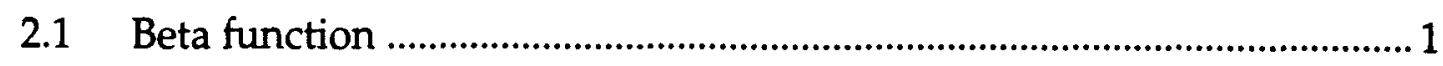

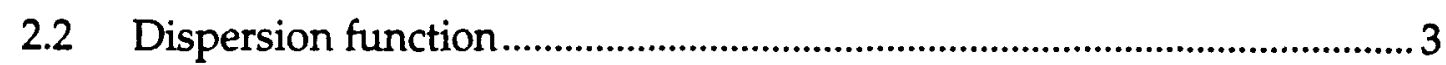

2.3 Matching the 8-GeV line vertical dispersion ..............................................

2.3.1 Dilution factor due to the mismatch................................................. 4

2.3.2 The matched vertical dispersion function .........................................4

2.3.3 Tunnel work expected ........................................................................

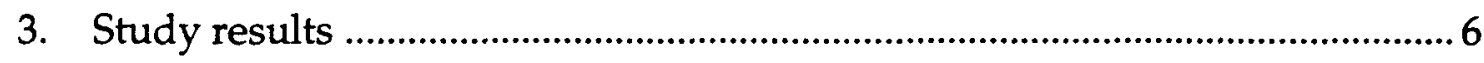

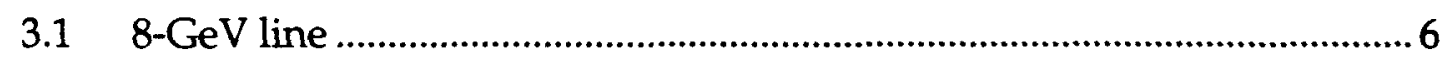

3.1.1 Dispersion function ......................................................................

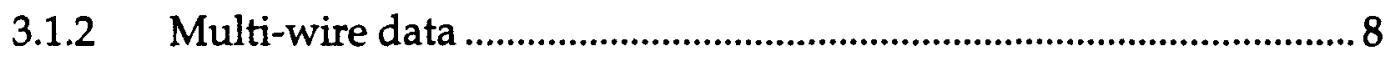

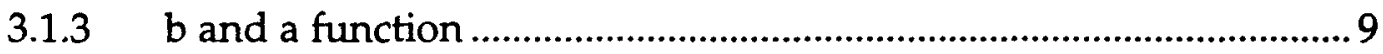

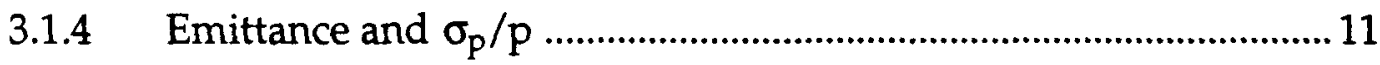

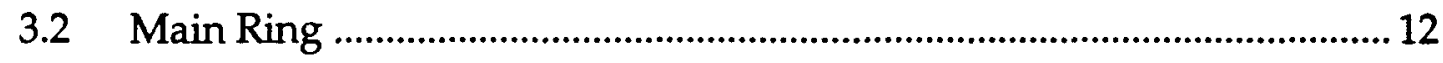

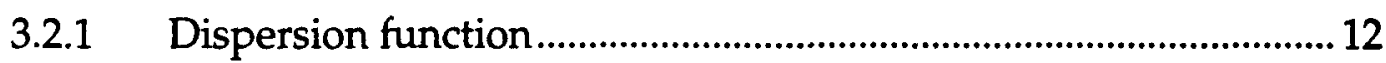

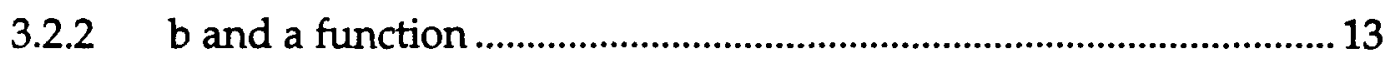

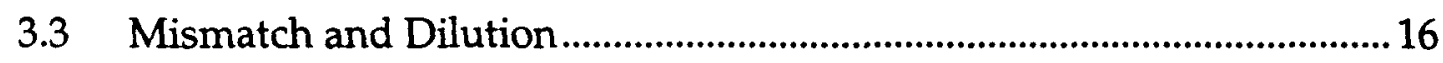

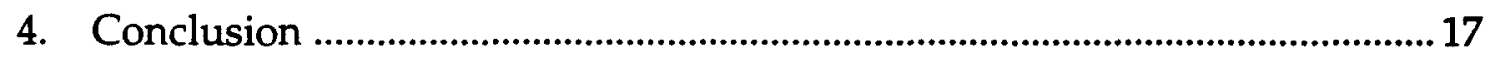

Appendix A: Dilution due to 8-GeV line dispersion mis-match ............................ 19

Appendix B: Dilution due to Beta function mis-match ............................................ 20 


\title{
The 8-GeV Transfer Line Injection into MainRing
}

\author{
Ming-Jen Yang, AD/MAD
}

\section{Introduction}

Included in this report are a brief review of the design lattice of the $8-\mathrm{GeV}$ beam transfer line and the Main Ring, the recent measurements on the 8-GeV line lattice function as well as that of the Main Ring at 8-GeV.

The $8 \mathrm{GeV}$ transfer line was re-designed by M. Syphers in 1987 [1]. Since then it has been left more or less the same except occasional tuning by the control room operators. An effort was made to document and improve the $8 \mathrm{GeV}$ line during run 1a, at around March of 1993 and at the beginning of run 1b. A series of studies were conducted to extract the beta and dispersion function of the 8-GeV beam line, and the dispersion function of the Main Ring (MR). The Main Ring beta function measurement is a separate effort and is not covered in this report. The information obtained from the studies is needed to ascertain the transfer line to be at its best possible state.

The injection matching is a very important part of the MR operation. Mismatches such as energy, timing, or position are easily corrected because they cause oscillations which are visible on the Turn-By-Turn (TBT) TV monitor display. Mis-matches due to beta and dispersion functions are detected only by using the Flying Wire or by doing measurements during beam study. A new method which makes use of the available data from TBT hardware was used to obtain the beam phase space ellipse. Data taken from Main Ring at injection gives the beta function needed for transfer matching from $8-\mathrm{GeV}$ line. The result of this measurement is also presented here.

\section{Design lattice function}

\subsection{Beta function}

The horizontal transfer line beta function from Booster and into Main Ring is shown in figure 1(a) while the Main Ring beta function starting at $A \varnothing$ is shown in 1(b). The vertical transfer line beta function is shown in 2(a) and the Main Ring vertical beta function is shown in figure 2(b). The beta function of the $8 \mathrm{GeV}$ line is well matched in either plane. 

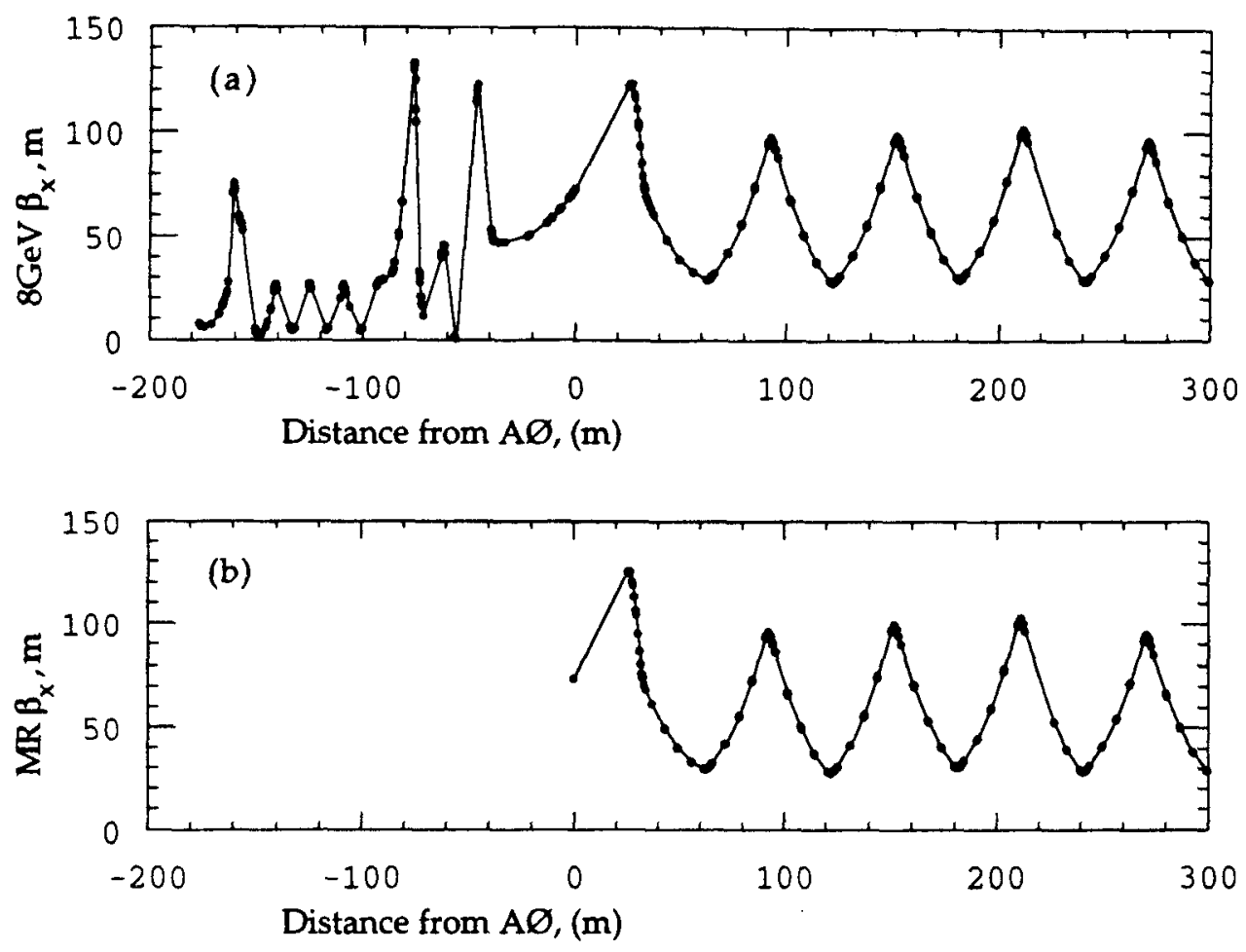

Figure 1. Horizontal beta function from SYNCH calculation. (a) is for $8-\mathrm{G} \theta \mathrm{V}$ transfer line and (b) for Main Ring A-sector.
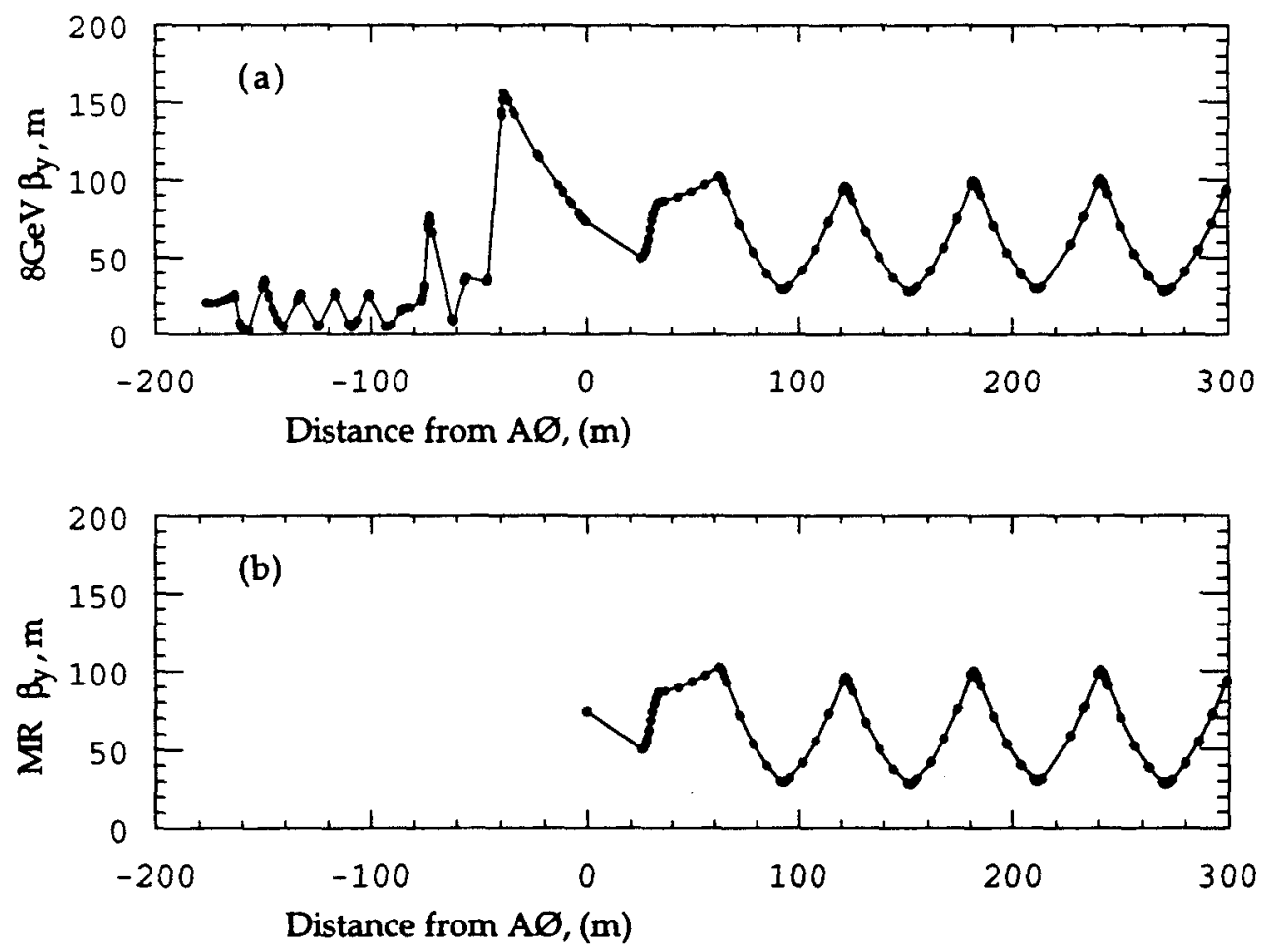

Figure 2. Vertical beta function from SYNCH calculation. (a) is for $8-\mathrm{GeV}$ transfer line and (b) is for MR A-sector. 


\subsection{Dispersion function}

The horizontal dispersion function of the transfer line is shown in figure 3(a) and that of the Main Ring in figure 3(b). The match is again near perfect. The vertical dispersion functions of the transfer line is shown in figure 4(a) and the MR vertical dispersion function in 4(b). It is quite clear that the vertical dispersion function is not matched.
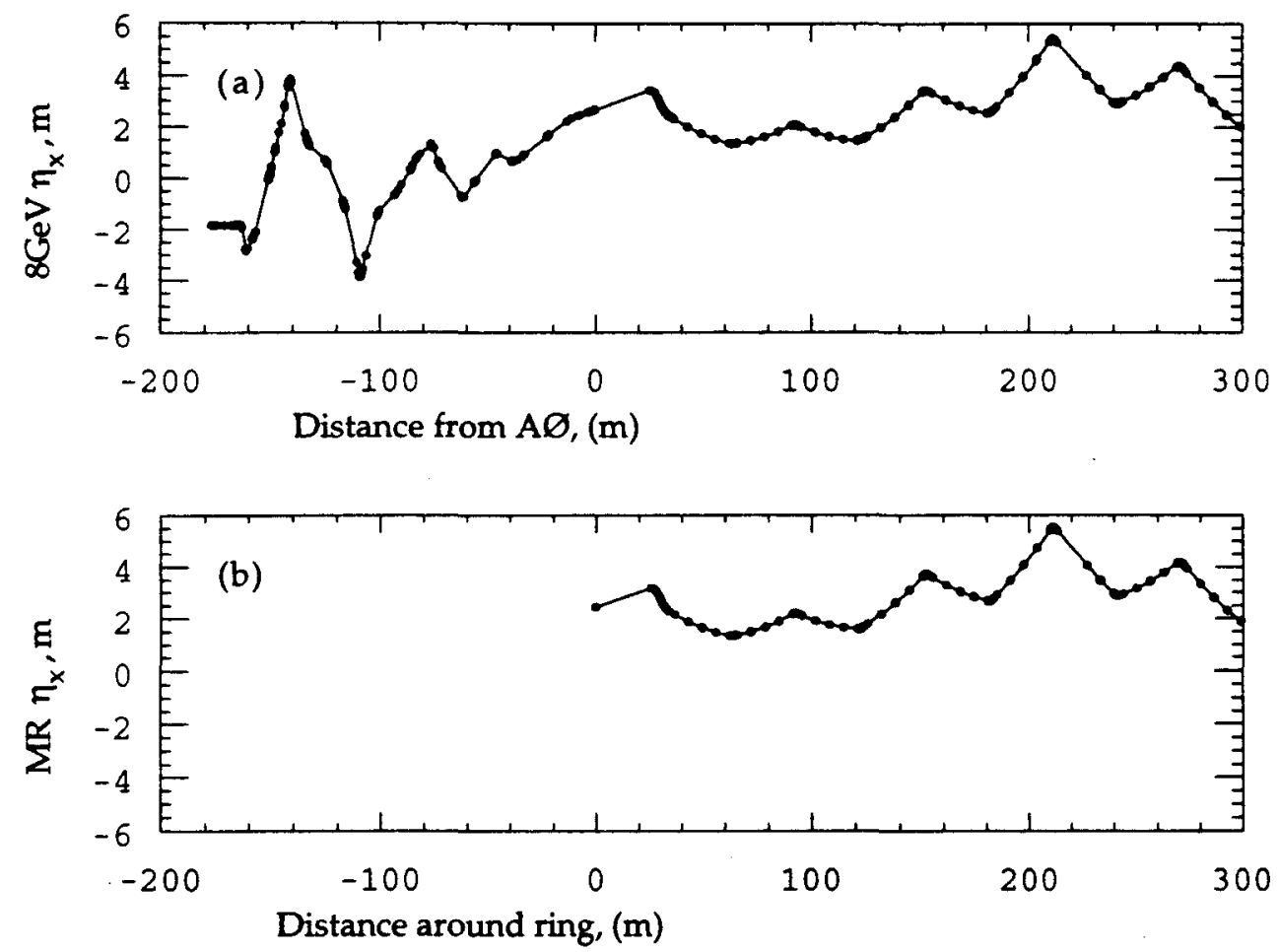

Figure 3. Horizontal dispersion function from SYNCH calculation. (a) is for 8-GeV transfer line and (b) for Main Ring A-sector.

\subsection{Matching the 8-GeV line vertical dispersion}

The mis-match in the design vertical dispersion function is likely the result of the $1988 \mathrm{D}$ Ø over-pass dispersion upgrade. The $8-\mathrm{GeV}$ transfer line was commissioned just a year before and its design dispersion function was matched to the Main-Ring at the time. There has been no attempt to re-match the dispersion function since the overpass upgrade. 

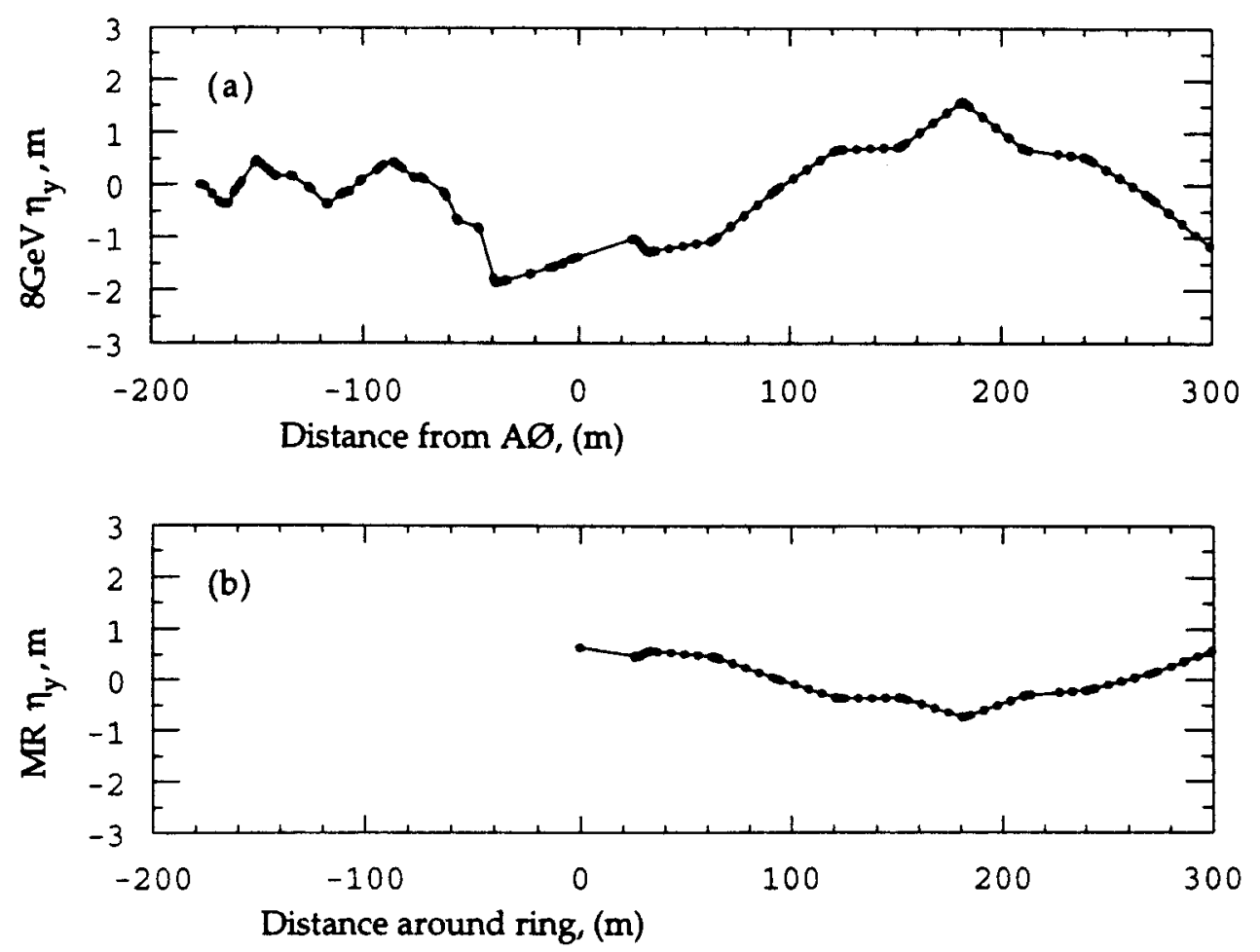

Figure 4. Vertical dispersion function from SYNCH calculation. (a) is for $8-\mathrm{GeV}$ line and $(b)$ for the Main Ring A-sector.

\subsubsection{Dilution factor due to the mismatch}

The emittance dilution as a result of vertical dispersion function mis-match is estimated is shown in Appendix A and is between 5 and $10 \%$. for the current beam emittance from the Booster.

\subsubsection{The matched vertical dispersion function}

The matching of the vertical dispersion function can be done with no change to the beta function in either plane and the horizontal dispersion function as well. Figure 5 shows the modified 8-GeV line vertical dispersion function, to be compared with figure 4. A good match is again achieved.

\subsubsection{Tunnel work expected}

\section{Beam line}

The horizontal layout of the beam line will remain exactly unchanged. The vertical beam line layout is shown in figure 6. The new line is shown with connected solid line and the existing line in connected dashed lines. All the magnets and instrumentation between VTB1 and VTM 2 will have to be moved downward by amount necessary. 

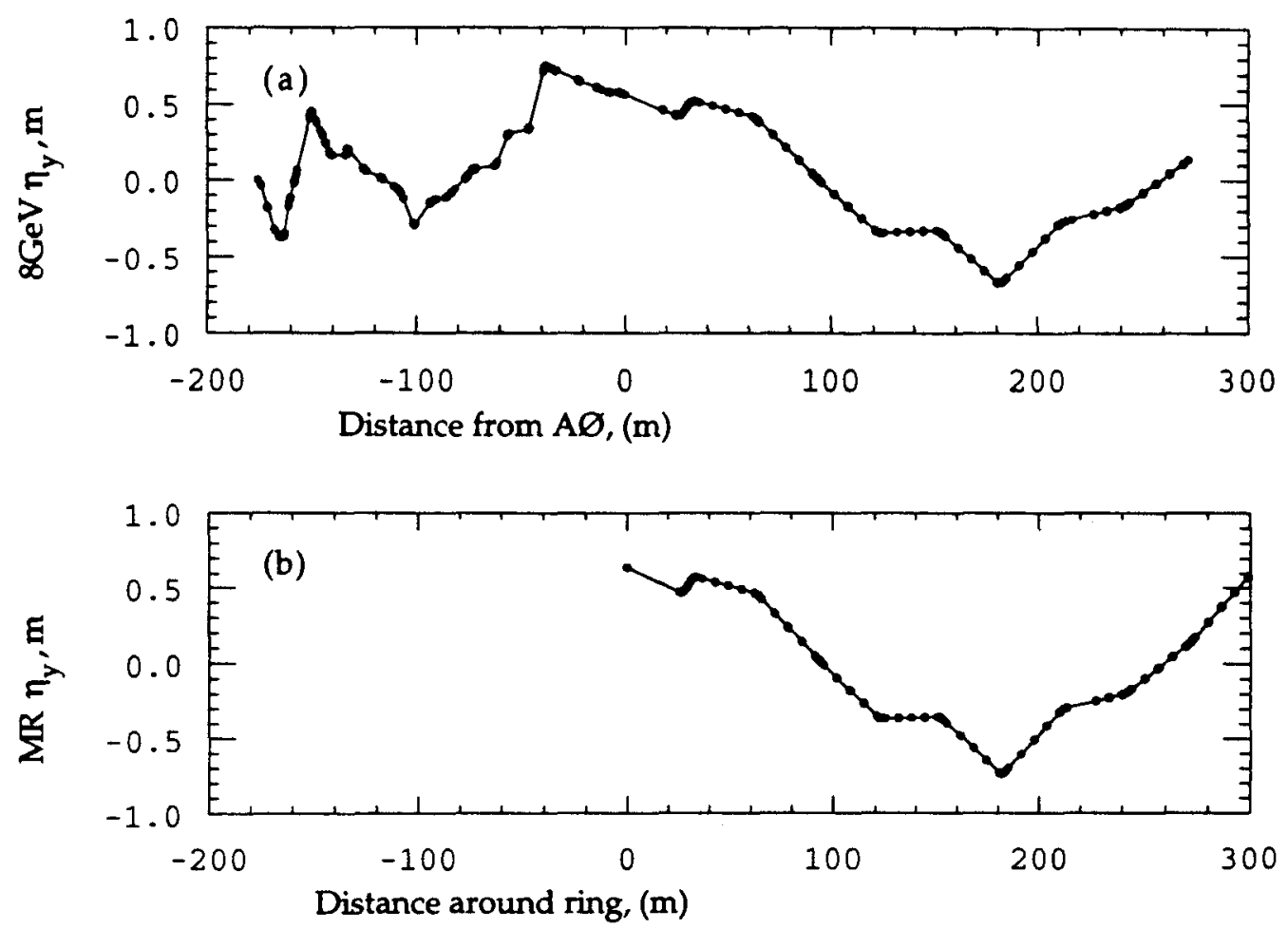

Figure 5. The modified 8-GeV transfer line for matching the vertical dlspersion function with that of the Main Ring. The plot scale range has been changed for finer display resolution. The Main Ring portion of the display is identical with that of the figure 4.

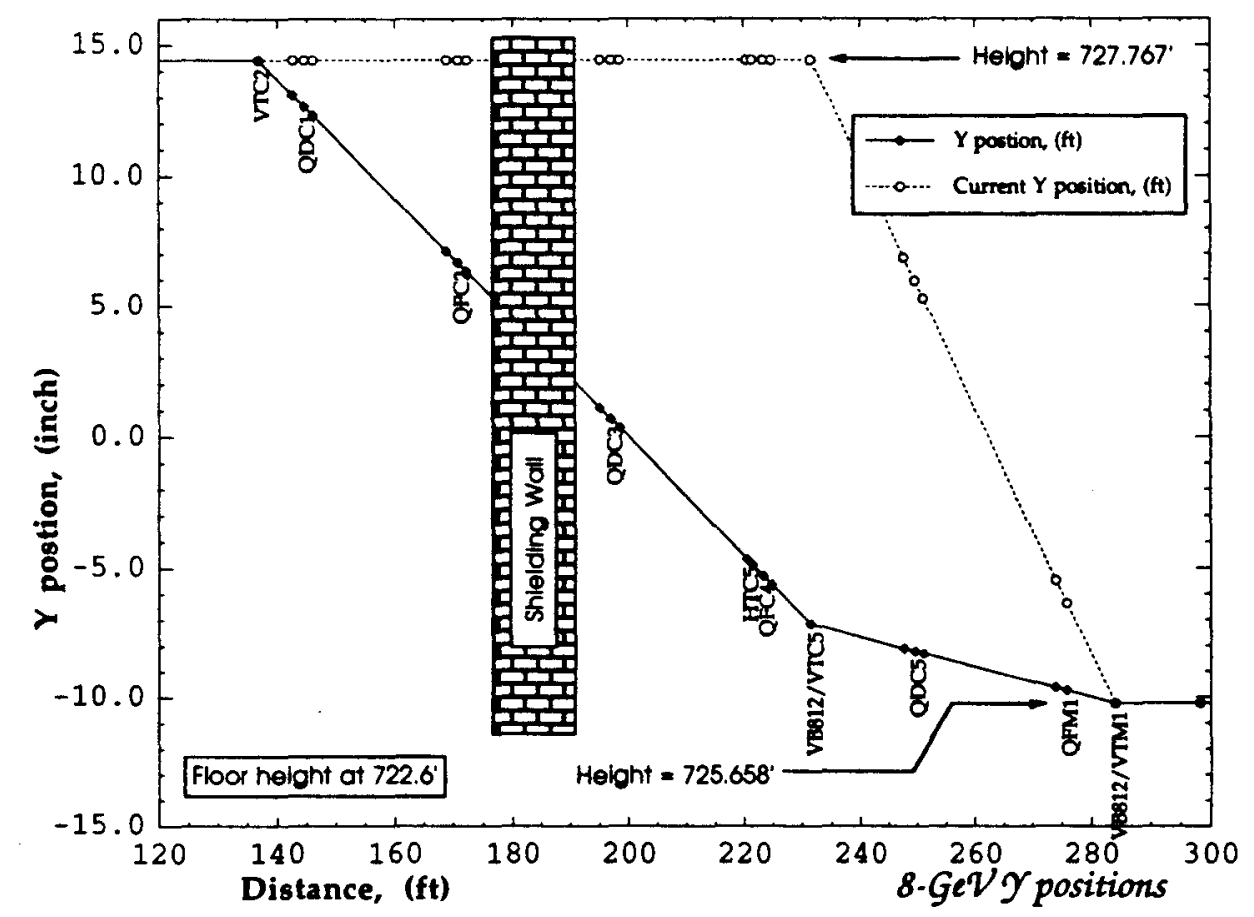

Figure 6. Modification on the $8-\mathrm{GeV}$ beam line positions to achleve vertical dispersion function matching to that of the Main Ring. The horlzontal positions remain unchanged. 
TM-1923

\section{Shielding wall}

Since the beam line will start to slope down before the shielding wall a new penetration will need to be made and the old be plugged.

\section{Magnets}

The modification involves changing the 8-GeV line vertical dog-leg from two magnet combination into a three magnet combination of VTC2, VTC5/VB812 and VTM1/VB812. VTC2 will be putting out a downward kick of about $19 \mathrm{mr}$ instead of being near zero. VTC5 will have an upward kick of about $14 \mathrm{mr}$ instead of a downward kick of $21 \mathrm{mr}$. VTM1 will have about $5 \mathrm{mr}$ of upward kick instead of $21 \mathrm{mr}$. One possibility would be to replace the VTC2 magnet with the one at VTM1 and replace the VTM1 magnet with a smaller magnets.

\section{Study results}

The studies were done both at later part of run 1a and at the beginning part of run $1 b$. While in run 1a the emphasis was more on documenting and understanding the state of the transfer line. In run $1 \mathrm{~b}$ the purpose was to verify what was learned during the previous run.

\subsection{8-GeV line}

\subsubsection{Dispersion function}

The $8-\mathrm{GeV}$ line dispersion function was measured by taking $8 \mathrm{GeV}$ transfer line beam positions at various Booster output energy. The Main Ring to Booster phase lock loop was disabled. The Booster radial feedback loop was kept on to ensure that the beam orbit in the Booster remained unchanged. This made the Booster horizontal dispersion function at the extraction location to be effectively zero. The $8-\mathrm{GeV}$ line stayed tuned to a fixed $8-\mathrm{GeV}$ energy during the study. 


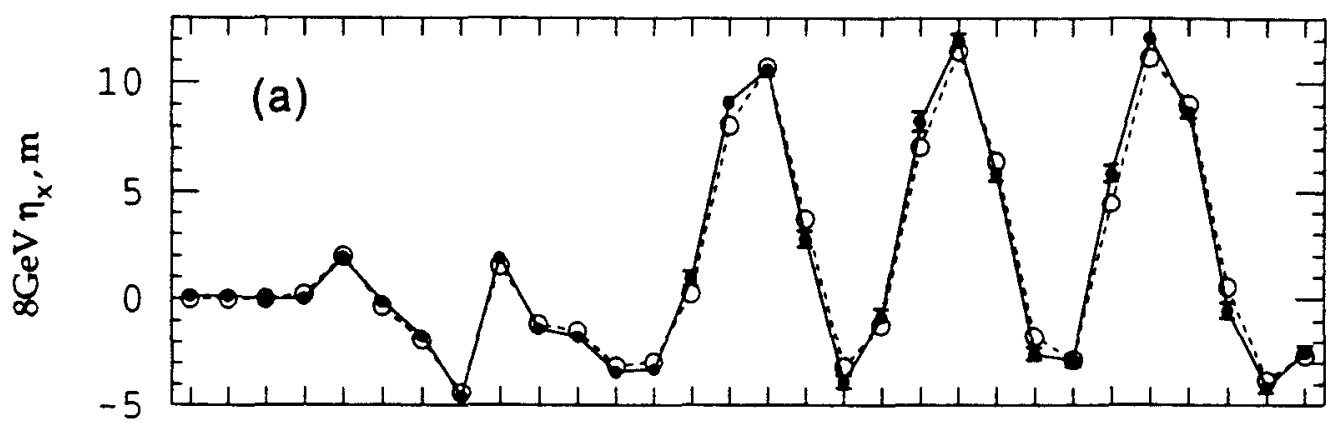

ન્-

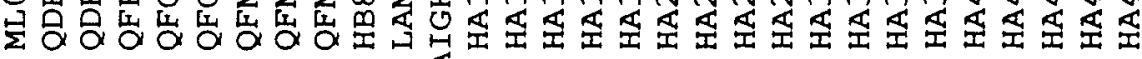

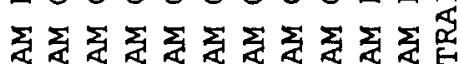

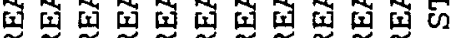

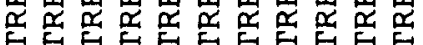

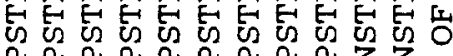

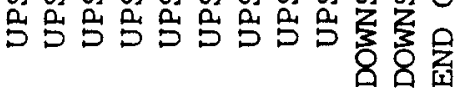

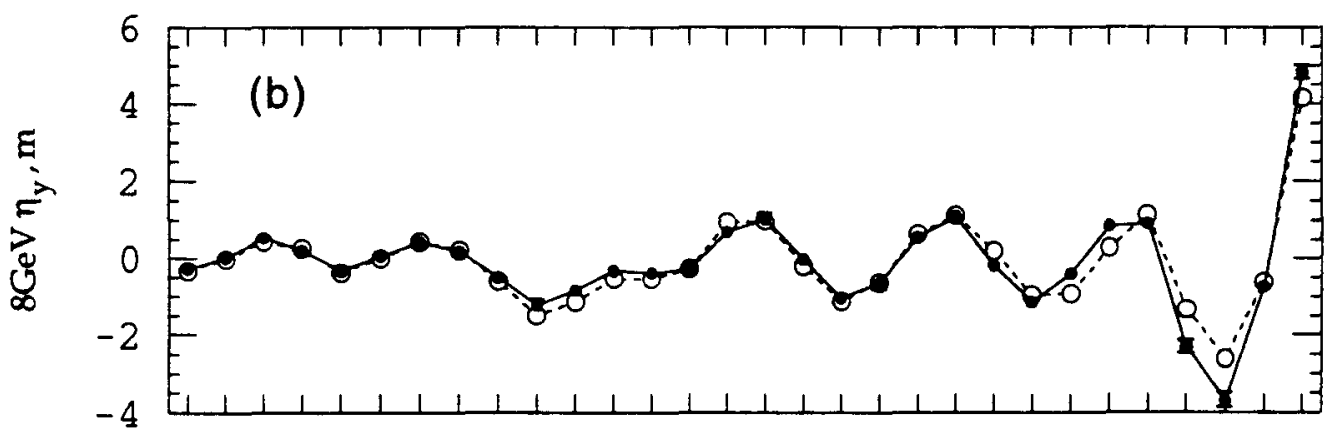

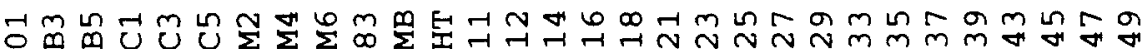

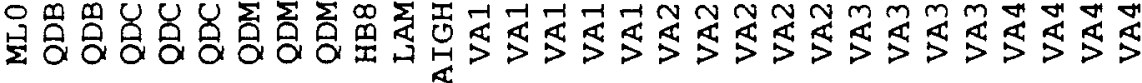

$\sum \sum \sum \sum \sum \sum \sum \sum \sum \sum$

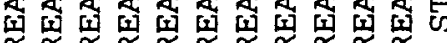

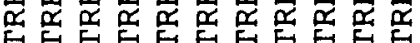

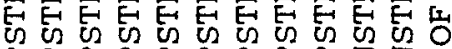

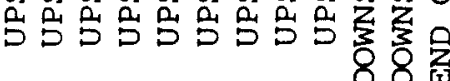

Figure 7. Measurement of 8-GeV transfer line dispersion function on 4/06/93.

The open circles are from SYNCH calculation and solld dots are measured data with error bars. (a) is for the horizontal dispersion function and (b) for vertical.

Booster device B:IMAX was used to control the output energy. The percent change in the IMAX is taken to be the $\Delta \mathrm{p} / \mathrm{p}$ in beam momentum [2]. A linear fit to the beam position as a function of $\Delta p / p$ was performed to derive the measured dispersion function at each BPM location. Figure 7(a) and 7(b) show the horizontal and vertical dispersion function as measured in April 1993. Figure 8(a) and 8(b) shows the same measurement done in October 1993. In both figures the SYNCH calculation based on the beam line setup at the time are shown in connected dash lines. Because the $8-\mathrm{GeV}$ beam was aborted on first turn into the 
Main Ring the transfer line actually ends at B49. Figure 7 and 8 displays BPM data only up to A49.

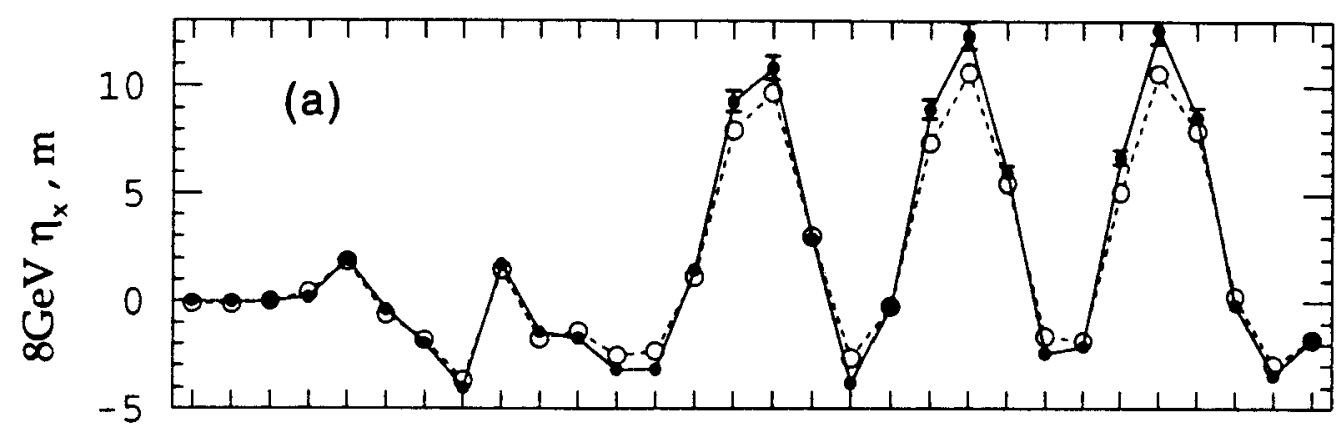

$m$ m

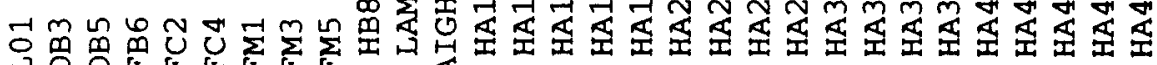

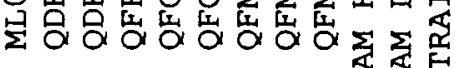

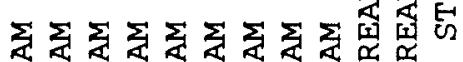

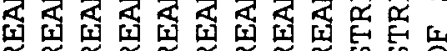

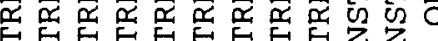

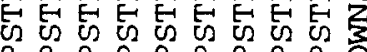

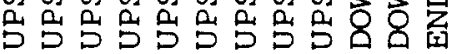

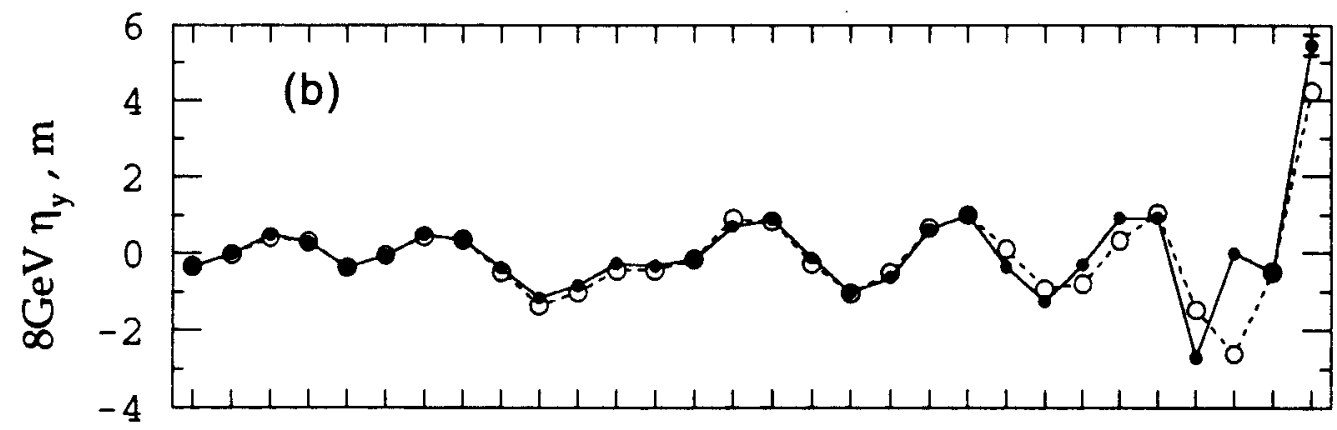

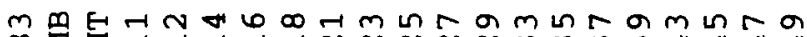

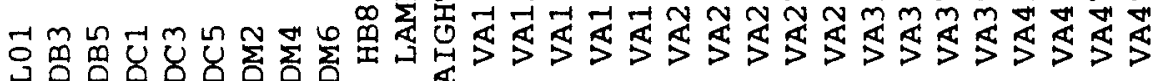

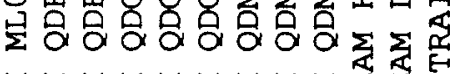

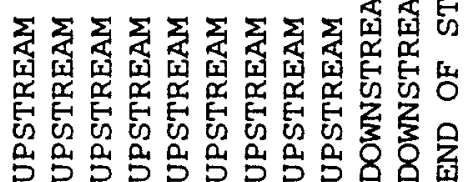

Figure 8. Same as in figure 7 but with measurement data obtain on 11/12/93.

\subsubsection{Multi-wire data}

The 8-GeV line multi-wire data is used to obtain Gaussian beam profile sigma. This calculation is done on-line by the C192 CAMAC card processor and can also be done by console program M45. The profile data is assumed to have a Gaussian distribution. The fitting algorithm of the console program has several advantages over that of the $\mathrm{C} 192$ micro-processor. It is less susceptible to error due to missing wires within the profile or to missing portion of the profile when beam is off-centered. It is also more immune to background noise. It also has the 
ability to get input on the range of fitting from user to get around any potential bad fit. Difference between the two calculated result is typically around $10 \%$ or more. Figure 9 shows an example of the multi-wire data at WBO. Shown in solid black dots are the data, the solid line represents the fit to a Gaussian distribution by the M45 console program and the dashed line by the C192 micro-processor.

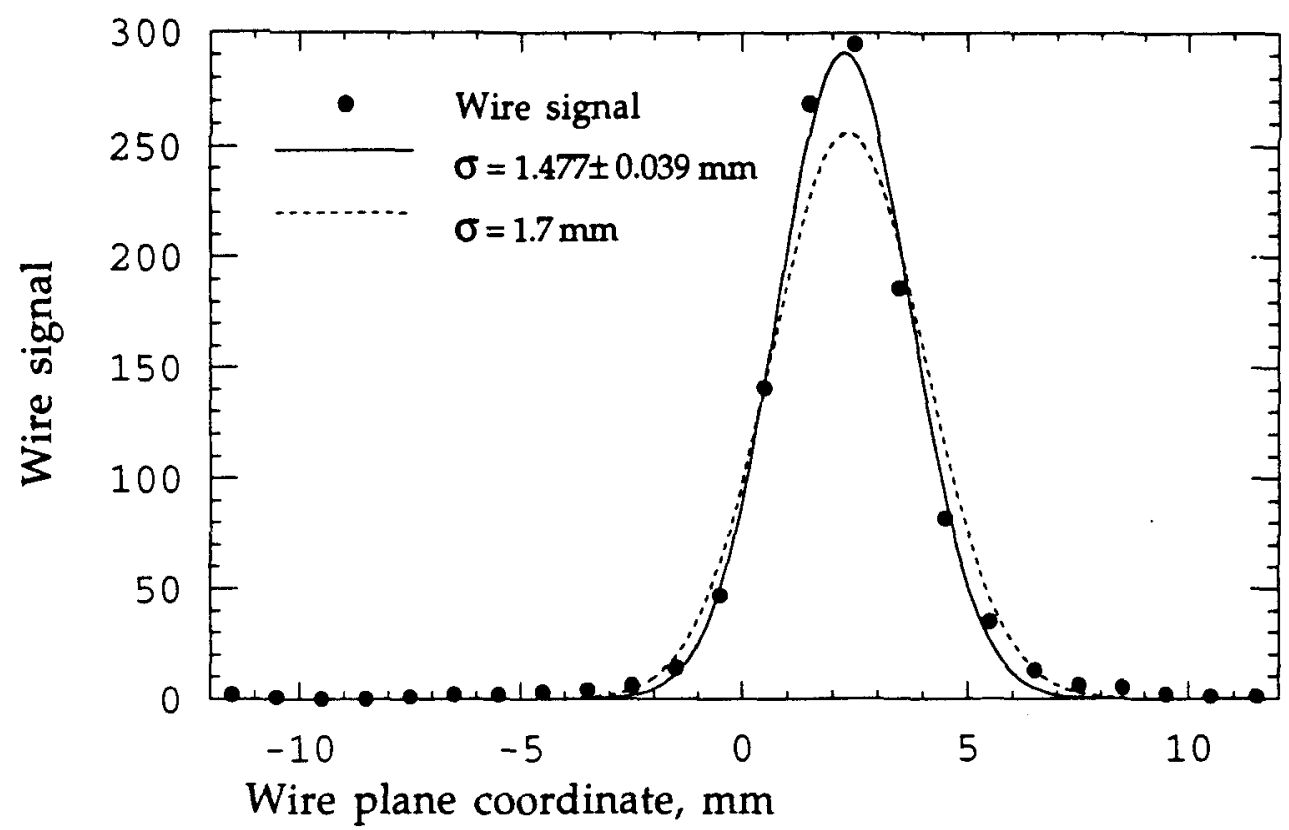

Figure 9. Multi-wire profile of WB $\varnothing$. There are total 24 wire in the plane. The solid curve is the Gaussian fit done by console program M45 and the dashed line by $\mathrm{C} 192$ micro-processor.

\subsection{3 $\beta$ and $\alpha$ function}

The sigma's obtained from multi-wire data are used in the fit for the beam emittance $\varepsilon$ and its $\sigma_{p} / p$. The fitting procedure uses the $\beta$ and $\alpha$ function values which were taken from the SYNCH calculation. The goodness of the fit gives an indication of how well the lattice function calculated by SYNCH can reproduce the data. Figure 10 shows the beam profile sigma from multi-wire data taken in April 1993 in solid black dots. Also shown are two sets of expected profile sigma. The open circles with connecting dotted lines are profiles calculated based on the beam line magnet setting at the time of study. The open diamonds are profiles calculated based on the design of M. Syphers. In either case the lattice function as calculated by SYNCH was used to fit for the $\varepsilon$ and $\sigma_{\mathrm{p}} / \mathrm{p}$ first and the expected wire profile sigma calculation follows. Again, (a) is for horizontal and (b) for vertical plane. The multi-wire locations most sensitive to lattice function devia- 
tion are in the Main Ring and most of those Main Ring multi-wires were not operational at the time of the first study.

In November 1993 the beam line magnet settings was reset back to the original design of M. Syphers. This had become possible after a beam pipe misalignment at the down-stream of the injection Lambertson was corrected. The mis-alignment was found to be well over half an inch lower than what it should be vertically. This also explains why the operating tune of the $8-\mathrm{GeV}$ line had a lower beta function at injection over that of the Syphers', according to the SYNCH calculation,
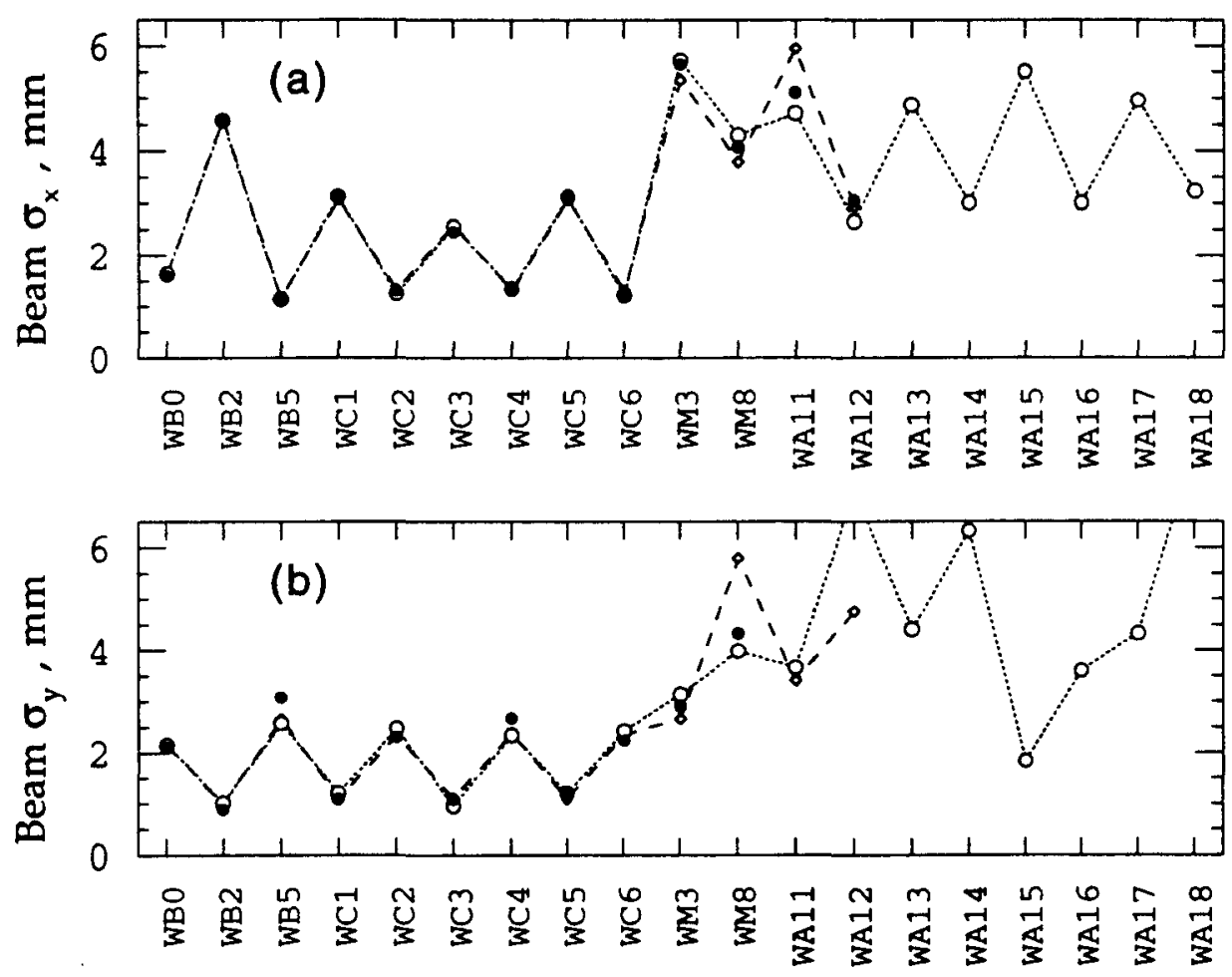

Figure 10. Multi-wire profile sigma as measured. The solld dots are the data. The open circles with connecting dotted lines are the values from the calculation based on the beam line magnet setting at the time of study. The diamonds with connecting dashed lines are values from calculation based on the design of $M$. Syphers.

The data taken in March 1994 with all multi-wire working is shown in figure 11 in the same way. The $8-\mathrm{GeV}$ line tune did not stray away by much at the time of this study. In fitting for $\varepsilon$ and $\sigma_{\mathrm{p}} / \mathrm{p}$ only the multi-wire data starting from $W B \varnothing$ and down to WC6 were used. The data and calculated profile agreed quite well in general. Since multi-wires down stream of WC6 were not used in the fit the good agreement at this portion of beam line is viewed as a positive confirma- 
tion. The estimated errors on the measured profile sigma are shown as vertical error bars in the figure.
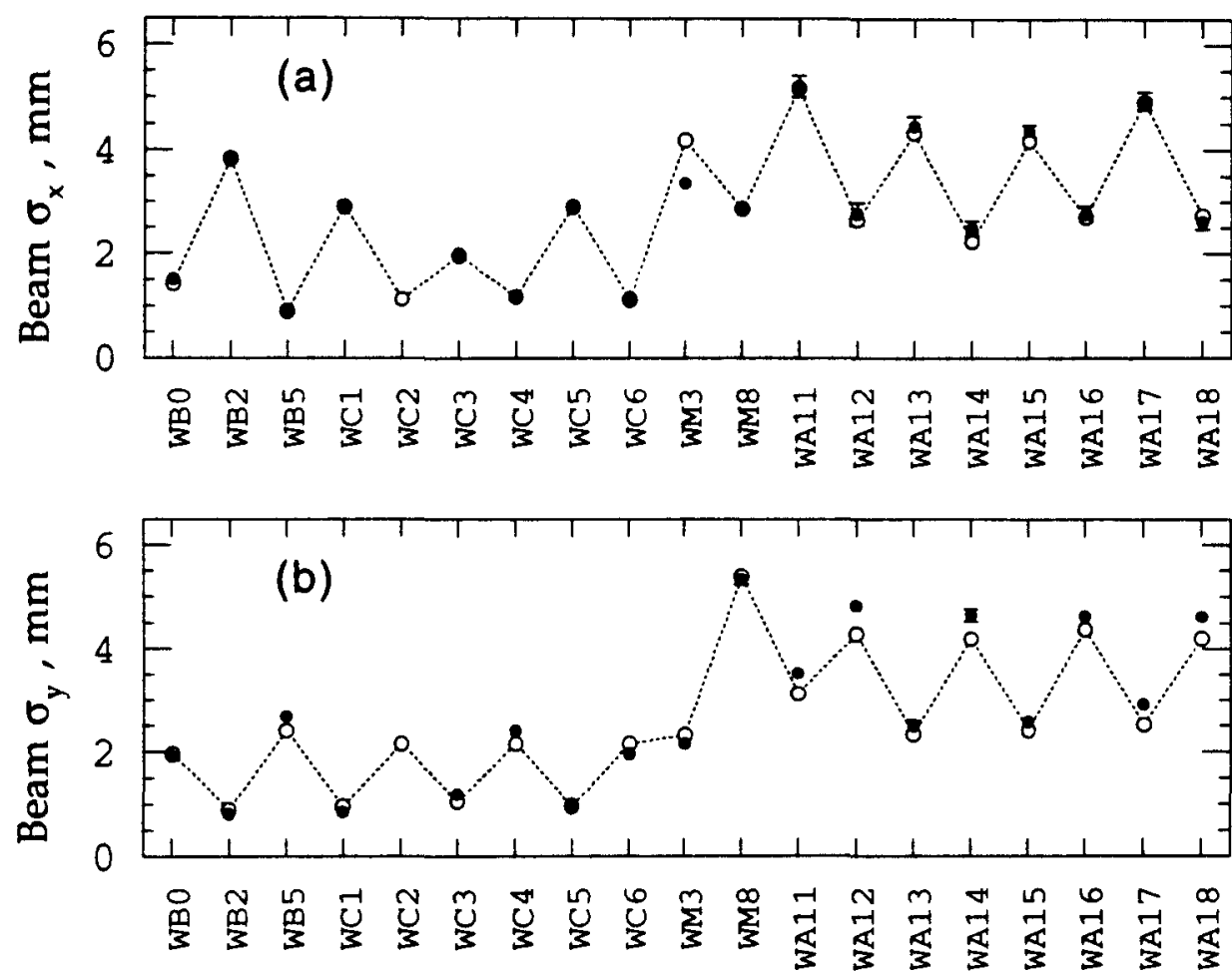

Figure 11. Multi-wire profile sigma as in figure 10 with data taken on $3 / 04 / 94$. The solid black dots are data with error bars and open clrcles are the calculated sigma using fitted emittance and $\sigma_{p} / p$.

The Booster extraction point lattice function used for the calculation appears to be in good agreement with data. Future possibility would be to actually fit for the Booster extraction lattice function when the beam line transfer matrix can be verified to the appropriate accuracy.

\subsubsection{Emittance and $\frac{\sigma_{p}}{p}$}

One of the design feature of the $8-\mathrm{GeV}$ line is that it can be used to analyze the horizontal and vertical plane emittance and as well as the $\sigma_{\mathrm{p}} / \mathrm{p}$. In March 1994, well after the start-up of the new $400 \mathrm{MeV}$ Linac, the multiwire data was taken with Booster beam at 2 and up to 8 turns. The beam intensity as a function of number of Booster turns is shown in figure 12(a). The emittances of both horizontal and vertical plane and $\sigma_{\mathrm{p}} / \mathrm{p}$ are shown in figure 12(b). 
TM-1923
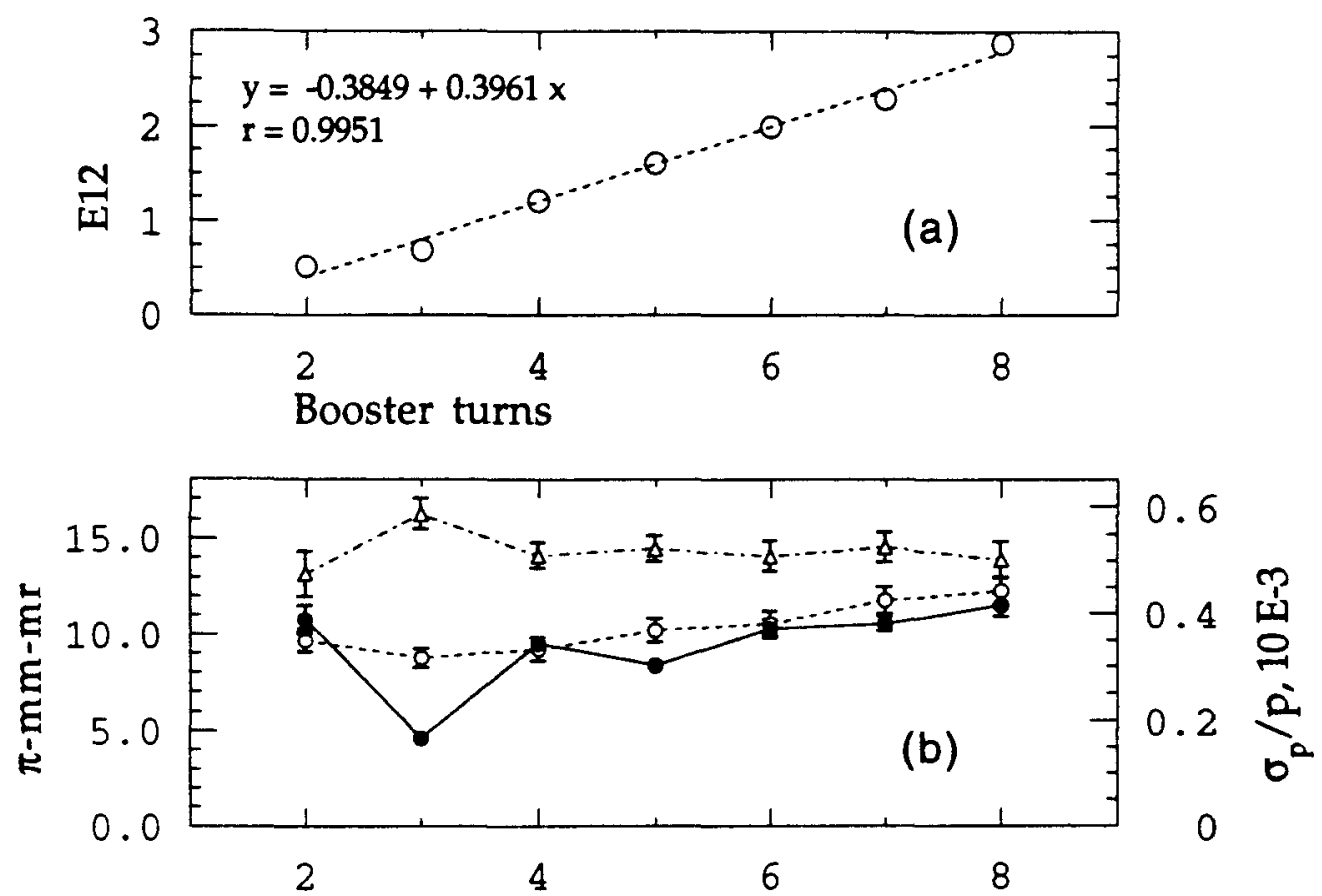

Booster turns

Figure 12. (a) The $8-G e V$ beam intensity entering Main Ring as a function of number of Booster turns. (b) Beam emittance as a function of number of Booster turns. The scale for the horizontal emittance (solid dots) and vertical emittance are shown on the left hand side of the plot and the scale for $\sigma_{\mathrm{p}} / \mathrm{p}$ (triangles) is shown on the right hand side of the plot.

For comparison the emittance during the April 1993 study, with 6 Booster turn beam at $\sim 2 \mathrm{E} 12$, the horizontal emittance was $14.9 \pm .4 \pi-\mathrm{mm}-\mathrm{mr}$, the vertical emittance was $12.6 \pm .13 \pi$, and $\sigma_{\mathrm{p}} / \mathrm{p}$ was $0.48 \pm .02 \times 10^{-3}$.

\subsection{Main Ring}

\subsubsection{Dispersion function}

Two studies were done to measure the MR dispersion function. One is in April of 1993, before the June 1993 shut-down and the other in October after the New $400 \mathrm{MeV}$ Linac came on line. The measurement done in April was flawed because the information on the beam momentum was not recorded. The analysis has to rely on using HF28 BPM data to provide $\Delta p / p$ information to fit for the dispersion function. This presents a real problem at least in the absolute calibration on the values of dispersion function.

In the October study the Main Ring RF frequency was recorded and used to calculate the $\Delta \mathrm{p} / \mathrm{p}$ information. The result of the measurement is shown in figure 13(a) for horizontal plane and 13(b) for vertical plane. The data is in solid cir- 
cle with error bar and SYNCH calculation of the Main Ring lattice function in open circle with connecting dashed lines. To most extent the result agrees well with the SYNCH calculation.

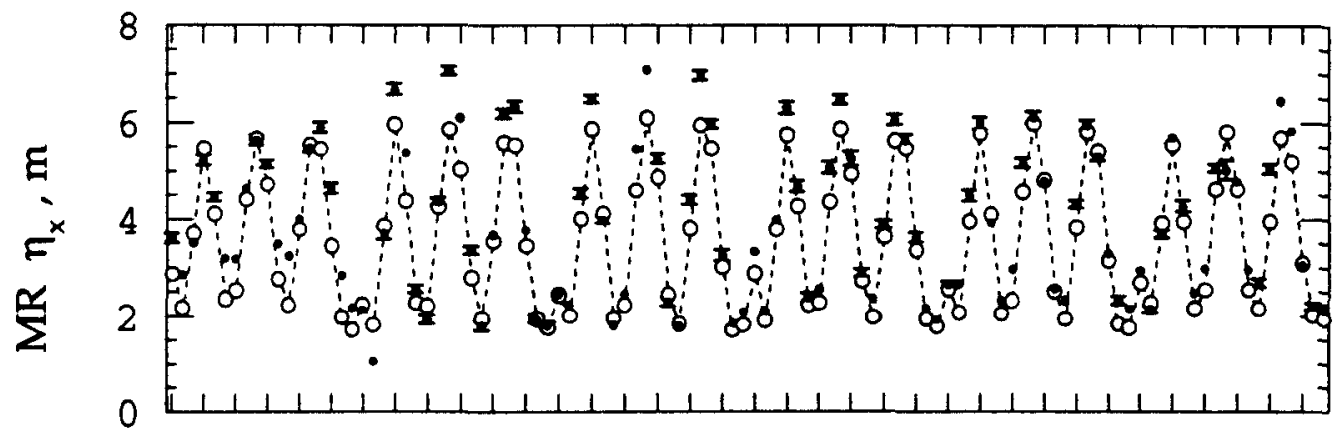

HT

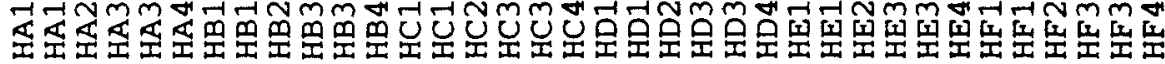

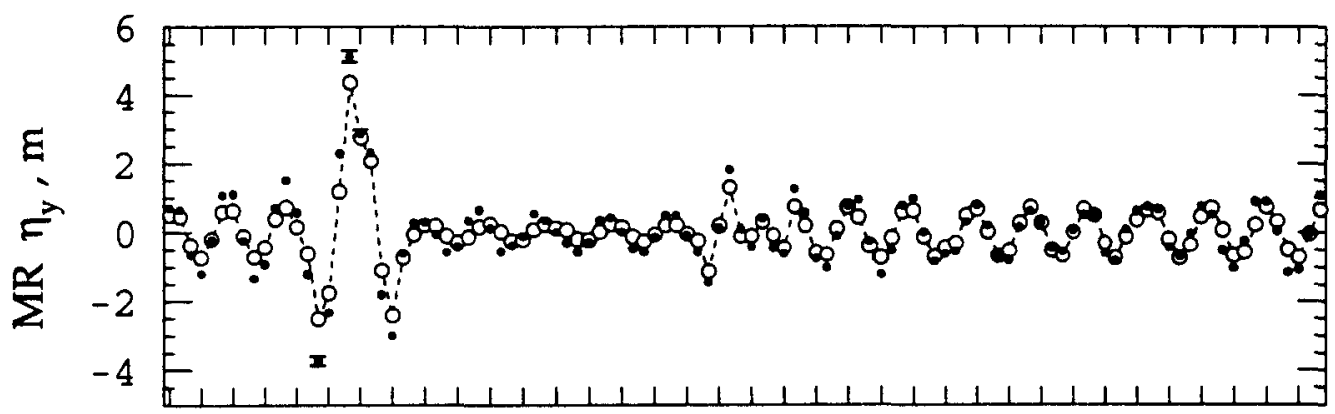

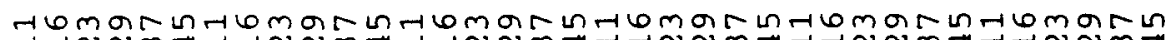

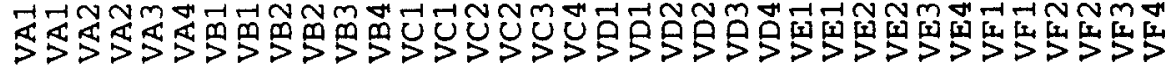

Figure 13. Measured Main Ring dispersion function at injection. The solld dots with error bars are the data and the open circles with connecting dashed lines are the SYNCH calculated values. (a) is for horizontal plane and (b) for vertical plane.

There appears to be some modulation on the horizontal dispersion function around the Main Ring and the measurement tend to be higher than that of the design calculation. For the vertical dispersion function the agreement is much better.

\subsection{2 $\beta$ and $\alpha$ function}

The beta and alpha function for the Main Ring is not as easily measurable. The traditional way to measure the beta function is to excite correctors in the ring and to record changes in closed orbit. Depending on the method of calculation this will require prior knowledge of either the phase advances between correctors and BPM's or the tune of the machine. Effort had been made to do just that [3]. 

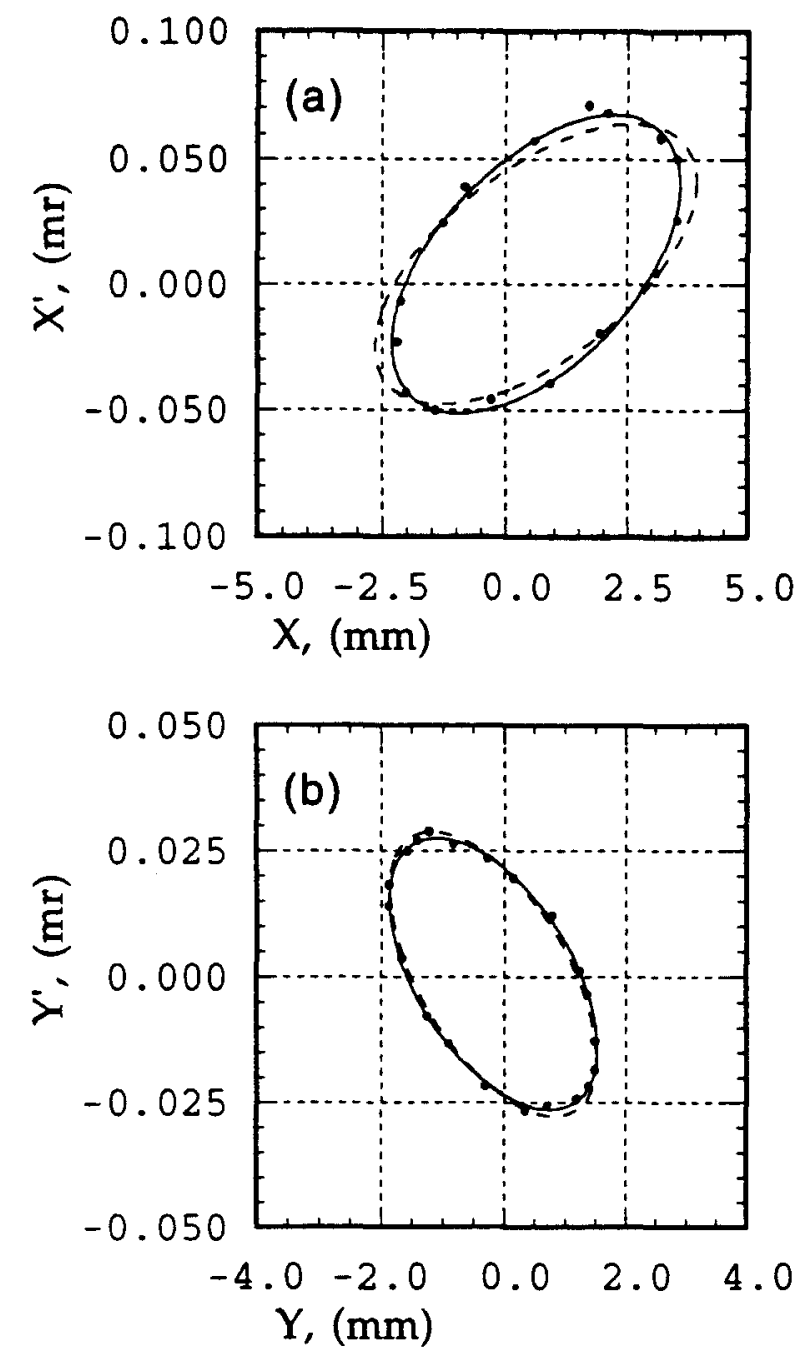

Beta: $\quad 59.11 \quad(73.51) \mathrm{m}$ Alpha: $-0.6437(-0.7458)$

Tune: $-0.436 \pm .0016$
Beta: $\quad 75.07 \quad(73.98) \mathrm{m}$ Alpha: $0.6483(0.7364)$ Tune: $-.4195 \pm .0011$

Figure 14. Main Ring phase space plot at location $A \varnothing$. The solid dots are the measured data points. The solld ellipse is drawn based on the fitted beta and alpha values. The dashed line ellipse is based on the designed Main Ring lattlice function whose values are shown inside the parenthesis.

For the purpose of injection matching to Main Ring we will need to know only the beta and alpha at one location in the Main Ring, preferably between the injection Lambertson magnet and the MR A-sector multiwires. A logical choice would be at AØ. The MR Turn-By-Turn BPM hardware provides information which can be used to derive beam phase space coordinates such as $\left(x, x^{\prime}\right)$ or $\left(y, y^{\prime}\right)$ for every turn of the beam. During an excited betatron oscillation these beam phase space coordinates will form an ellipse which can be fitted to get the values of beta and alpha [4].

Figure 14(a) is the phase space plot of the fitted $x$ and $x^{\prime}$ for turn number 5 to 20 at Main Ring location AØ. The fitted ellipse is shown in solid curve with its 
associated $\beta$ and $\alpha$ values shown in the lower right corner. The values from SYNCH calculation are shown enclosed in the parenthesis with its corresponding ellipse drawn in dashed line. Slight differences between the measurement and the calculation values are visible. The tune of the machine can be fitted using the TBT angles in the normalized phase space and is displayed along with the lattice function. Exactly the same thing for vertical plane is shown in figure 14(b). The agreement between measurement and calculation is quite good in the vertical plane.

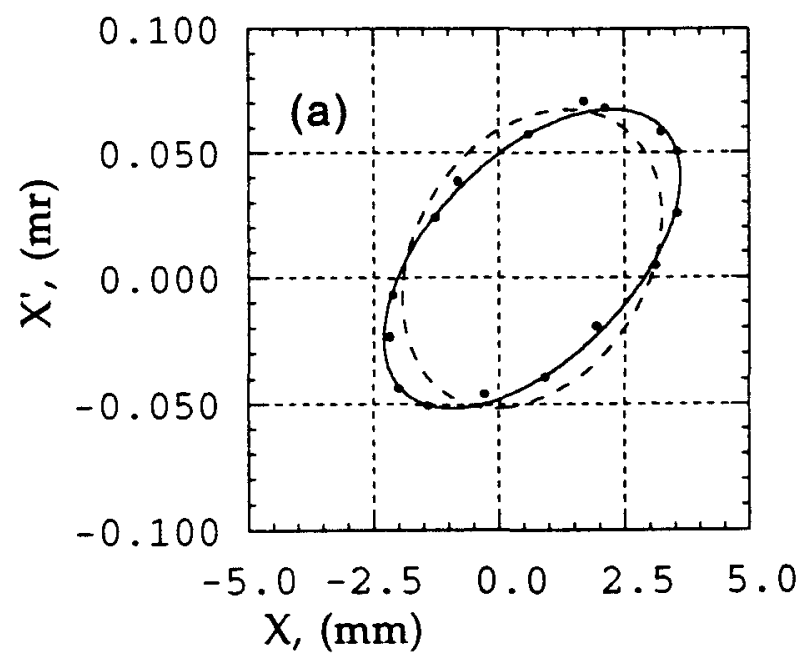

Beta: $\quad 59.11 \quad(45.252) \mathrm{m}$

Alpha: $-0.6437 \quad(-0.2872)$

Tune: $-0.436 \pm .0016$

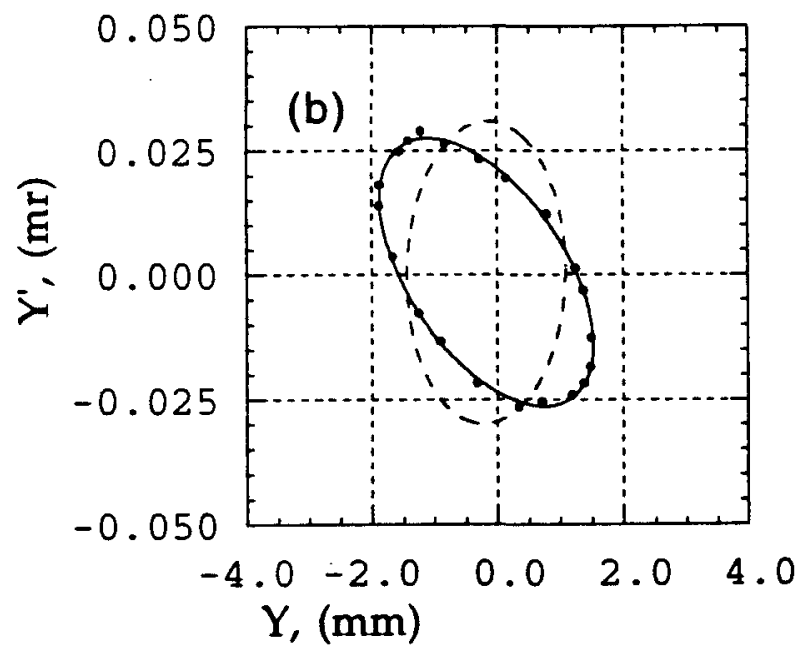

Beta: $\quad 75.07 \quad(41.639) \mathrm{m}$ Alpha: $0.6483 \quad(-0.0345)$

Tune: $-0.4195 \pm .0011$

Flgure 15. Main Ring A $\varnothing$ phase space ellipse as compared with the expected injection phase space ellipse. Both the solid dots and the solid ellipse are the same as those of figure 14. The dashed ellipse drawn based on the injection lattice function whose values are shown inside the parenthesis, as of $4 / 06 / 93$. 


\subsection{Mismatch and Dilution}

Based on the results from the April 1993 study the likely 8-GeV line lattice function did not fit the Main Ring very well. Figure 15 shows the same plot as in figure 14 except that the data is shown against the expected injection beta function at $A \varnothing$ instead of the calculated Main Ring lattice function. The amount of mis-match is quite shocking. Shown in figure 16 is the beta function and in figure 17 the dispersion function, as of April 1993. The lattice function along the transfer line is very different from the Syphers' design as shown in Fig. 1 - 4. The analysis indicated that the amount of dilution could be around $20 \%$ in horizontal plane and $50 \%$ in vertical plane, see appendix $B$.

The results of dispersion function measurements agree well with calculation. The vertical dispersion function mismatch was confirmed as expected and its effect on dilution is already covered earlier in this report.
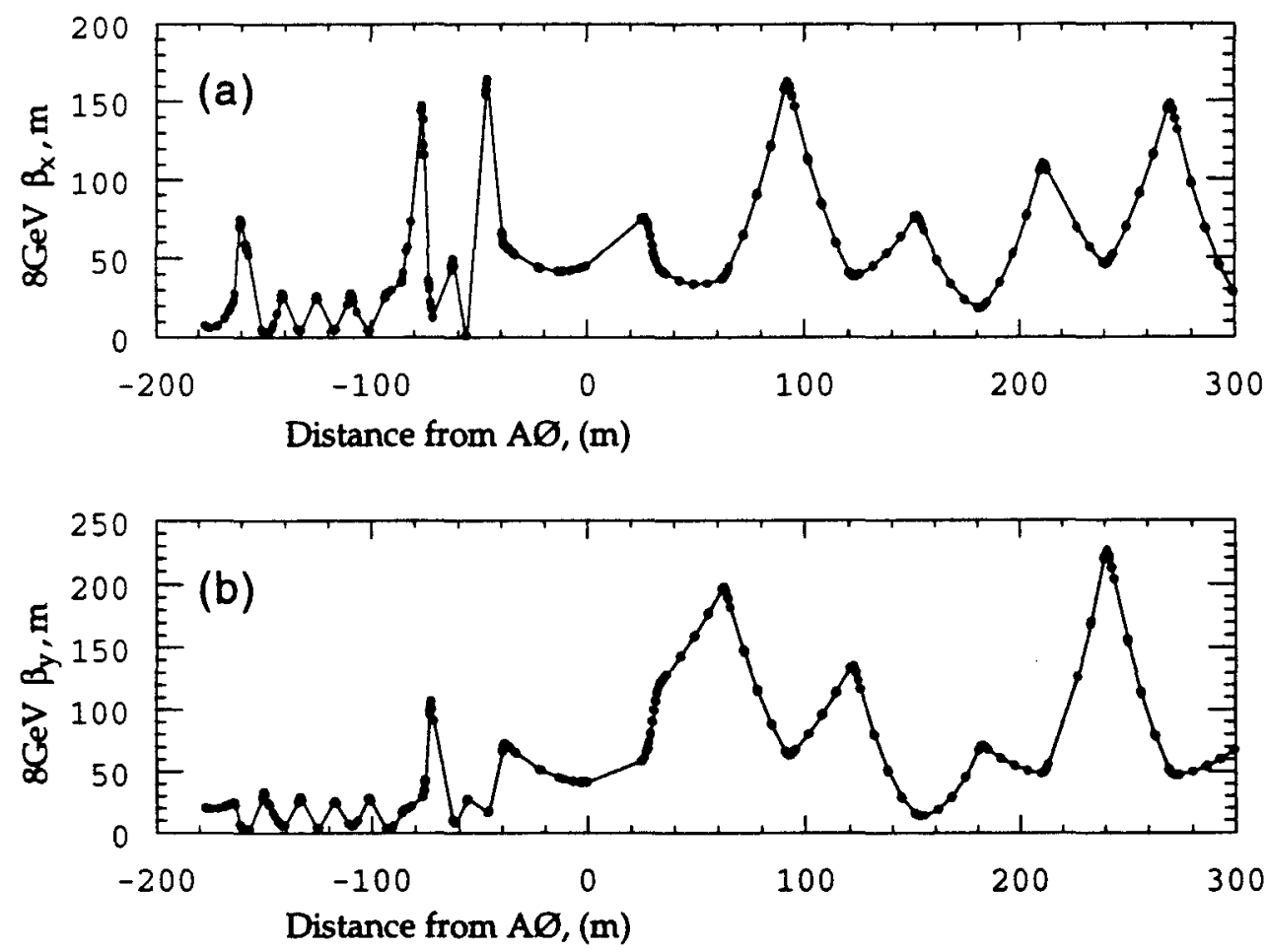

Figure 16. The expected beta function for the 8-GeV transfer line as of $4 / 06 / 93$.

(a) is for the horizontal beta function and (b) for vertical beta function. 

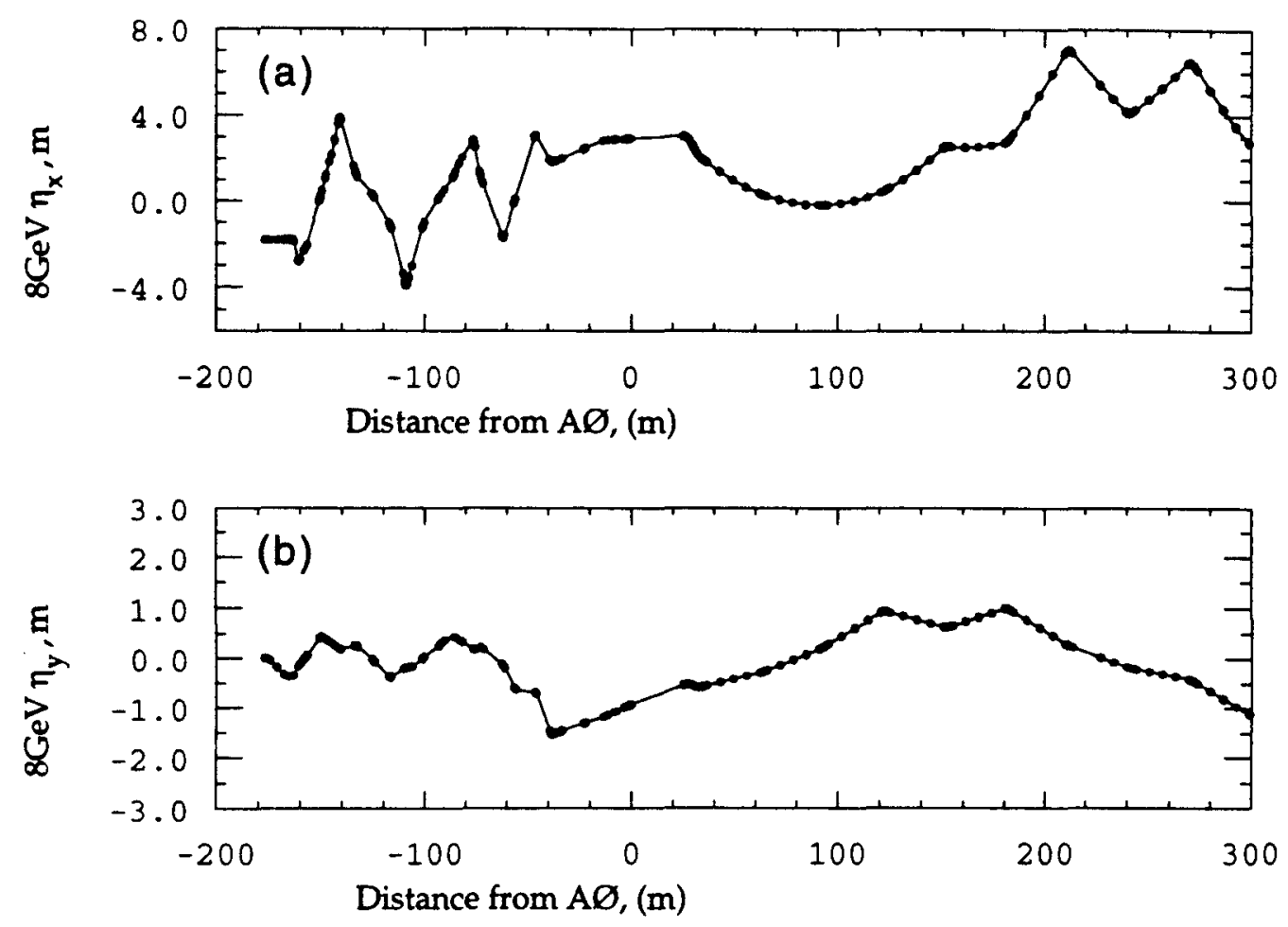

Figure 17. The expected eta function for the $8-\mathrm{GeV}$ transfer line as of $4 / 06 / 93$.

(a) is for the horizontal dispersion function and (b) for vertical dispersion function.

\section{Conclusion}

The result of study indicated that the readings on the quadruple magnets gives an acceptable description of the beam line lattice function. The $8-\mathrm{GeV}$ line magnet settings had wondered away from the design values over the years, most likely as a result of the mis-aligned beam pipe. At the conclusion of the study, all 8-GeV line quads were left to the setting consistent with magnet gradients in the original design of M. Syphers. This should optimize the injection matching of the $8-\mathrm{GeV}$ line to the designed Main Ring lattice. The resulting comparison of phase space ellipses between the injected beam and the Main Ring circulating beam is shown in figure 18.

The Turn-By-Turn data is very useful in addressing the issue of the $8-\mathrm{GeV}$ line injection matching to the Main Ring. It is conceptually straight forward and operationally much simpler. The traditional way of measuring lattice function by observing orbit distortion caused by dipole corrector magnets takes quite a bit more effort. The discrepancy in the Main Ring horizontal $\beta$ function will have to be resolved. 

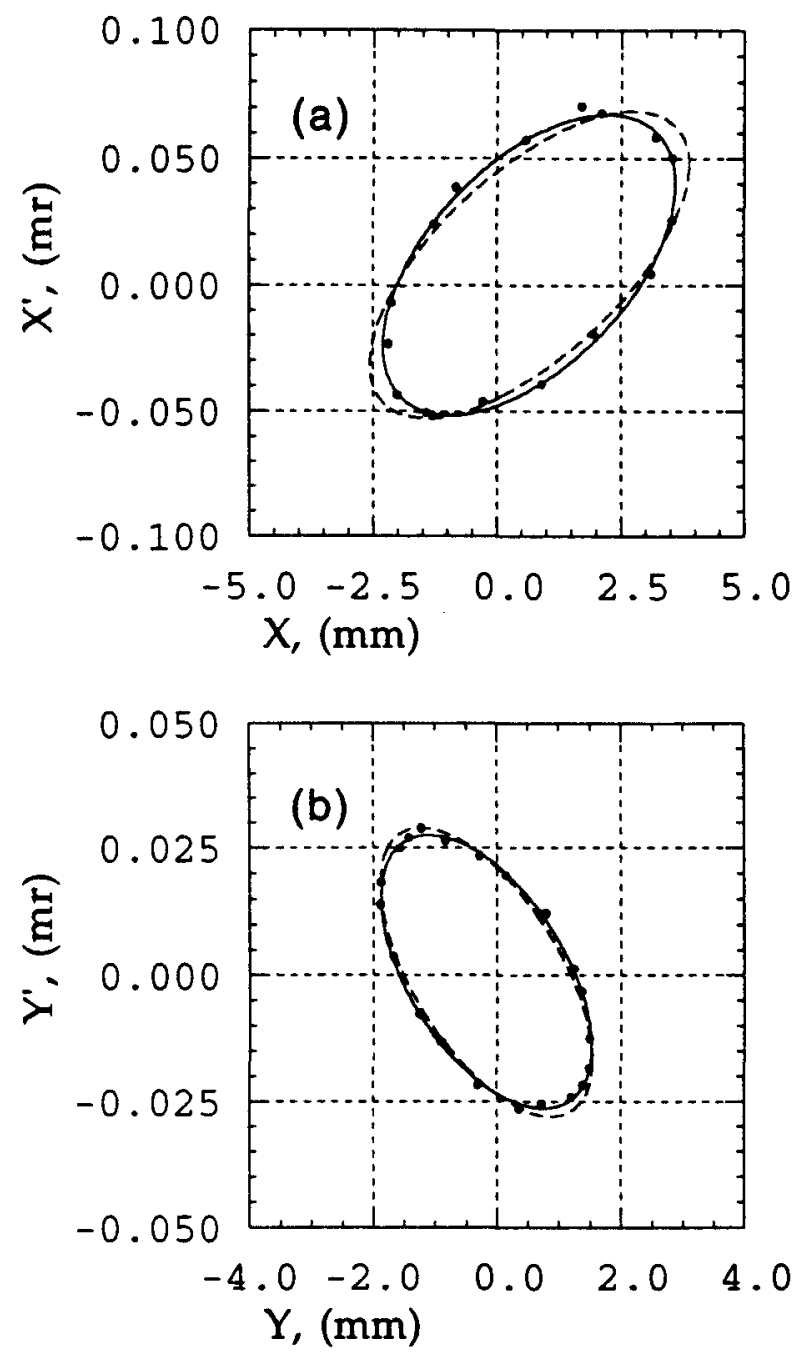

Beta: $\quad 59.11 \quad(70.0479) \mathrm{m}$

Alpha: $-0.6437 \quad(-0.86557)$

Tune: $-0.436 \pm .0016$
Beta: $\quad 75.07 \quad(75.9032) \mathrm{m}$ Alpha: $0.6483 \quad(0.77483)$ Tune: $-0.4195 \pm .0011$

Figure 18. The comparison of Injection and MainRing phase space ellipse as of March 1994. The 8-GeV line magnet settings was restored in November of 1993 to give the designed quadrupole field gradient.

1 M. Syphers, "An Improved $8 \mathrm{GeV}$ Beam Transport System for the Fermi National Accelerator Laboratory", FERMILAB TM-1456, 1987.

2 Within this document, the $\Delta \mathrm{p}$ is defined as the deviation of the average beam momentum from the reference while $\sigma_{p}$ is the width of the Gaussian distribution in the momentum variation within the beam particles.

3 Q. Wu, internal communications.

4 M.J. Yang, "Lattice Function Measurement with TBT BPM Data", Fermilab TM-1922 


\section{Appendix A: Dilution due to 8-GeV line dispersion mis-match}

Numbers

$$
\begin{aligned}
& \Delta \mathrm{D}=2 \text { meters } \\
& \sigma_{\mathrm{P}} / \mathrm{P}=0.0005 \\
& \beta \gamma \text { at } 8-\mathrm{GeV}=9.47 \\
& \varepsilon_{\mathrm{N}}=10 \times \mathrm{E}-6 \pi \text {-meter-radian } \\
& \beta=74 \text { meters }
\end{aligned}
$$

\section{Dilution factor}

Using the formula from the thesis paper by M. Syphers for the relationship for $95 \%$ emittance:

$$
\begin{aligned}
& 6 \pi \sigma^{2}=\beta \varepsilon=\frac{\beta \varepsilon_{\mathrm{N}}}{\beta \gamma} \\
& \sigma^{2}=\frac{\beta \varepsilon_{\mathrm{N}}}{\beta \gamma \times 6 \pi}=\frac{10 \pi \times \mathrm{E}-6 \times 74}{9.47 \times 6 \pi} \\
& \sigma=0.0036 \text { meters } \\
& \frac{\Delta \mathrm{D} \times \sigma_{\mathrm{P}} / \mathrm{P}}{\sigma}=\frac{2 \times 0.0005}{0.0036}=0.277
\end{aligned}
$$

From the Figure V-5 we could interpolate to see that the dilution factor will be about 1.05 . 


\section{Appendix B: Dilution due to Beta function mis-match} Numbers at $A \varnothing$

\begin{tabular}{|l|l|l|}
\cline { 2 - 3 } \multicolumn{1}{l|}{ Horizontal } & Beta & Alpha \\
\hline 8-GeV line & 45.2519 & -0.2872 \\
\hline Main Ring & 73.5026 & -0.7458 \\
\hline
\end{tabular}

\begin{tabular}{|l|l|l|}
\cline { 2 - 3 } \multicolumn{1}{l|}{ Vertical } & Beta & Alpha \\
\hline 8-GeV line & 41.6390 & -0.0345 \\
\hline Main Ring & 73.9618 & 0.7367 \\
\hline
\end{tabular}

\section{Dilution factor}

The formula to calculate the dilution due to beta function mismatch is given by Syphers as follows:

$$
\begin{aligned}
& F_{\beta}-1+\frac{1}{2}\left[\frac{(\Delta \beta / \beta)_{\text {eq. }}}{\sqrt{1+(\Delta \beta / \beta)_{\text {eq. }}}}\right]^{2}=D \\
& \text { where }(\Delta \beta / \beta)_{\text {eq. }} \equiv D+\sqrt{D^{2}-1}-1 \\
& \text { and } D=\frac{1}{2}\left[\beta_{1} \gamma_{2}+\beta_{2} \gamma_{1}-2 \alpha_{1} \alpha_{2}\right] ;
\end{aligned}
$$

\begin{tabular}{|l|l|l|l|}
\cline { 2 - 4 } \multicolumn{1}{c|}{} & $\Delta \beta / \beta$ & Dilution factor $F \boldsymbol{B}$ & From Figure V-7 \\
\hline Horizontal & 0.6996 & 1.144 & $\sim 1.2$ \\
\hline Vertical & 1.2541 & 1.349 & -1.55 \\
\hline
\end{tabular}

